\title{
"Women's Three Bodies": An Anthropological Perspective on Barriers to Safe Abortion Services in Kibera Informal Settlements, Nairobi, Kenya
}

\author{
Edna N. Bosire1), W.Onyango-Ouma ${ }^{2)}$ \\ ${ }^{1)}$ MRC/Wits Developmental Pathways for Health Research Unit (DPHRU), \\ School of Clinical Medicine, Faculty of Health Sciences, University of the Witwatersrand, \\ Johannesburg, South Africa \\ ${ }^{2)}$ Institute of Anthropology, Gender and African Studies, University of Nairobi, Nairobi, Kenya
}

\begin{abstract}
Background: Abortion remains a critical determinant of maternal morbidity and mortality in Kenya. Recent studies on induced abortion estimated an annual abortion incidence of 48 abortions per 1,000 women of reproductive age, which is higher than other East African countries. In 2010, the Kenyan Constitution widened the provisions under which women qualified for safe abortions. However, majority of women still seek abortion services from unqualified providers. Using an interpretive, meaning-centered approach, this study aims to explore the barriers to safe abortion services in Kibera informal settlements, Nairobi Kenya.

Subjects and Method: This was a cross-sectional study encompassing both qualitative and quantitative research methods. We used different sampling techniques (convenient, purposive and snow ball methods) to recruit our study participants $(n=55)$. Quantitative data was collected using a survey questionnaire and data was analyzed by SPSS version 21. Qualitatively, data was collected using Focus Group Discussions (FGDs), Key Informant interviews and case study narratives. This involved note taking and audio recordings, which were transcribed verbatim and thematically analyzed using QSR Nvivo 21 software.

Results: This study revealed that women's decisions to procure abortion are influenced by a myriad of complex factors: a multiplicity of meanings regarding abortion, as provided by the law or as dictated by cultures or religion. Women's access to safe abortion services were also determined by their ability to afford the procedure and to identify and reach a health care provider who offered the services.

Conclusions: Legal access to abortion does not simultaneously ensure access to safe abortion services. Policy makers and health promoters need to work towards changing the socio-economic and religious forces that hamper access to safe abortions. Safe and affordable abortion services to women in Kenya including post abortion care need also be ensured.
\end{abstract}

Keywords: barriers, access, safe abortion, Kenya

\section{Correspondence:}

Edna Nyanchama Bosire, MRC/Wits Developmental Pathways for Health Research Unit (DPHRU), School of Clinical Medicine, Faculty of Health Sciences, University of the Witwatersrand, Johannesburg, South Africa. 27 St Andrews Road, Parktown, Johannesburg, South Africa.

Email: edna.bosire@wits.ac.za. Telephone: 0117172383.

\section{BACKGROUND}

Unsafe abortions continue to be on the increase in Kenya despite the 2010 constitution widening the provisions permitting access to safe services (Jayaweera et al. 2018; Izugbara et al. 2017). The new constitution of Kenya (2010) explicitly permits abortion when "in the opinion of a trained health professional, there is need for emergency treatment, or the life or health of the pregnant woman is in danger, or if permitted by any other written law"(Centre for Reproductive Rights, 2010; National Council for Law Reporting, 2010). The 
constitution also states that "a person shall not be denied emergency treatment" (National Council for Law Reporting, 2010). However, despite the provisions in the law, desperate women continue to selfinduce abortions or obtain clandestine abortions carried out by untrained persons under poor hygienic conditions. Of concern is, why many women still procure unsafe abortions from unqualified providers, yet, the law provides for safe abortion services.

We argue that this scenario finds explanation in the concept of the 'mindful body' (Scheper-Hughes and Lock 1987) drawn from phenomenological theory. Phenomenology is the scientific study of experience and attempts to explain human consciousness in its lived immediacy (Jackson, 1996). Scheper-Hughes and Lock (1987) conceptualized the mindful body as constituting 'three bodies': individual bodyself, social body, and body politic. They defined the individual body as the "phenomenological sense of the lived experience of the body-self" (Scheper-Hughes and Lock 1987). Through the individual body "the patient's subjective experience" of abortion is realized, analyzed and comprehended. The social body perceives the body as a "natural symbol for thinking about relationships among nature, society and culture" (Scheper-Hughes \& Lock 1987). It is, regarded as a screen on which ideas of society, religion and culture are mapped. The body politic is a "regulation, surveillance, and control of bodies". It exerts a powerful control over all aspects of the individual body; its behavior, in reproduction and sexuality, in work, in leisure and in other forms of deviance and human differences" (Scheper-Hughes and Lock 1987).

In most countries, abortion remains one of the contested areas of debate and especially concerning its legalization and acceptability (Gleeson et al. 2008; Grimes et al. 2006; Cameron and Williamson 2005). These debates have been predominantly politicized and contested and may or may not include controversies about reproductive physiology, normative sexuallity, policies related to abortion, cultural and religious norms, demographic and political trends and family dynamics (Sawicki, 2011; Lopez, 2012; Siegel, 2014). As such, macro structures around the world, such as, the state have been dominant in deciding whether women are allowed formal access to abortion or not. The church, especially the Roman Catholic is well known for its stand on abortion: it equates it to murder since the life of a child begins at conception (Lopez, 2012). At least $26 \%$ of world citizens live in countries where abortion is prohibited by their governments (Anuradha et al., 2009). Prochoice and pro-life activists constantly dispute whether the woman has the ultimate say in the decision to abort or whether the right of life of the fetus should hold precedence (Lee, 2004; Lopez, 2012). Such standpoints are undoubtedly influenced by the larger political, religious, cultural, economic and moral considerations regarding abortion.

\section{Unsafe abortions in Kenya}

The magnitude of unsafe abortions in Kenya can be summarized by a recent headline in one of the Kenyan local newspapers which stated that; "Unsafe abortions kills seven Kenyans daily" (Daily Nation, 2018). Research shows that in Kenya, the incidence of induced abortion and maternal mortality from unsafe abortion is estimated to be much higher compared to other East African countries (Sedgh et al., 2016). A recent study on induced abortion estimated an annual abortion incidence of 48 abortions per 1,000 women of reproductive age (Ziraba et al., 2015), compared to an 
estimated annual abortion incidence of 34 per 1,000 in other East Africa region (Sedgh et al. 2016). As a result, over a third of the women with induced abortionsare admitted with abortion complications and are reported to be in the second trimester of pregnancy when risks of severe complications (such as sepsis, shock or organ failure) and mortality are substantially higher (Izugbara et al., 2017; Ziraba et al., 2015; Izugbara et al., 2015).

Until recently, the abortion law in Kenya was highly restrictive and only permitted abortion to save the life of the woman. The new constitution of Kenya (2010) provides stronger protection for the lives and health of women. It explicitly permits abortion when "in the opinion of a trained health professional, there is need for emergency treatment, or the life or health of the pregnant woman is in danger, or if permitted by any other written law" (National Council for Law Reporting 2010). Generally, the new constitution provides a new legal foundation for women's access to safe and legal abortion (Vélez, 2012). However, almost a decade later, women in Kenya still lack knowledge on the legal provisions for safe abortion as provided in the 2010 constitution, a factor that has driven women to procure unsafe abortions from unqualified providers (Hussain, 2012). Moreover, lack of clinical guidelines has led to ambiguity over the law's interpretation, and qualified providers remain untrained in the provision of safe abortion (Ziraba et al. 2015). Thus, safe and legal abortions are rarely practiced in the public setting, as both women and providers fear legal repercussions (Marlow et al. 2014; Centre for Reproductive Rights 2010).

Further, women's access to safe abortion services in Kenya is largely determined by their ability to access and afford the procedure (Jayaweera et al. 2018). Abort- ion related stigma has also been highly reported in Kenya and this has contributed to women seeking abortion services secretly from unqualified providers (Izugbara et al. 2017; Marlow et al. 2014), often utilizing unsafe methods including but not limited to: insertion of objects such as crochet needles or catheters into the uterus or ingestion of harmful substances such as herbs, medications, cleaners, and juices (Centre for Reproductive Rights, 2010; Izugbara et al., 2015; Mitchell et al. 2006).

Systemic challenges such as lack of equipment and the limited number of healthcare providers trained to perform abortions in Kenya also dramatically restricts women's access to safe abortion services and magnifies concerns about provider barriers to access, given that few providers can offer services (Centre for Reproductive Rights, 2010). Alarmingly, even for health care providers who have been trained to offer safe abortion services (including post abortion care)(Mayi-Tsonga et al. 2012), there have been reported cases of providers' violation and mistreatment of abortion care seekers including deliberately managing abortion patients without pain killers or threaten to have the police arrest them(Izugbara et al. 2015; Ndunyu 2013).This article applies an interpretive, meaning-centered approach to explore the barriers to safe abortion services in Kenya.

\begin{tabular}{l}
\hline \multicolumn{3}{c}{ SUBJECTS AND METHOD } \\
\hline 1. Study Design \\
This study was conducted in Kibera \\
informal settlement in Nairobi Kenya. \\
Kibera records high rates of sexual violence, \\
limited access to family planning, poverty \\
mean of 43 per cent and most pregnancies \\
are unwanted compared to other informal \\
settlements in the country (National \\
Coordinating Agency for Population and \\
Development, 2010). Majority of women
\end{tabular}


with unwanted pregnancies in Kibera often resort to unsafe abortions due to "illegallities" surrounding abortions and lack of access to safe abortion services (Izugbara and Ochako, 2011).

We employed a cross-sectional study design utilizing both qualitative and quantitative methods of data collection. Different sampling methods were used to recruit study participants. First, we conveniently recruited 50 women (aged 18-49) who participated in a survey questionnaire to gather their understanding of the constitutional provisions of abortion in Kenya, and factors influencing access to safe abortion services. The second stage entailed selection of focus group discussion (FGD) participants $(n=30)$ from women who had participated in the survey, based on their availability and willingness to participate in an FGD at a later stage. We conducted three FGDs (age stratified) as follows: older women (35-48), younger women (25-34) and girls (18-24). Each FGD comprised of 8-10 participants and took an average of two hours to complete. We also recruited eight (8) key informants for in-depth interviews using purposive sampling. They comprised of: Two (2) community health workers; three (3) health care providers; and three (3) religious leaders. The key informants lived and worked in Kibera. They were selected on the basis that they had interactedwith women who had procured an abortion or had a good knowledge on the topic of inquiry. Finally, five (5) cases, including those who had procured an abortion, were selected for this study using the snow ball technique. The cases participated in an in-depth interview where the participant was invited to tell her life history from puberty until the moment when the abortion occurred. During the conversation, we used probing questions to elicit the conversation as they narrated their experiences with abortion. Gradually, we added questions related to decisionmaking, help-seeking behavior, and general understanding on the barriers that women experienced in accessing safe abortion services in Kibera. All qualitative interviews and discussions involved note taking and audio recordings, which were transcribed verbatim.

\section{Data analysis}

Qualitative data was analyzed thematically usingQualitative Solutions for Research (QSR) NVivo 12 software. Transcripts and field notes were read and re-read by the first author to familiarize with the data and reviewed using a phenomenological approach to develop broad themes. This process was reviewed by the second author who individually reviewed the themes and immersed himself into the data to check and verify whether the themes correlated with the data. We then had a meeting where we discussed all the emerging themes and any identified discrepancies were resolved at this point. Together, we developed, tested, and refined a data codebook that was developed in Nvivo software for coding and analysis. Nodes were then applied to relevant blocks of text in the transcripts. Emergent new nodes were added to the codebook, and all transcripts were reviewed again in an iterative process. Nodes were summarized in analytical memos and verbatim excerpts were used to report the dominant themes; vignettes were generated from case study narratives to give an in-depth experience of the topic of inquiry. Quantitative data was analyzed by SPSS version 21. Data was entered in SPSS database where frequencies were generated, and cross-tabulations were used to examine the relationships between different variables. 


\section{Ethical Consideration}

We explained the purpose and objective of the study to participants, informing participants about voluntary participation and rights to withdraw from the study, seeking written informed consent, conducting the interviews in a private space and ensuring participants' information was kept confidential. The study was approved by the National Commission for Science Technology and Innovation (NACOSTI) in Kenya.

\begin{abstract}
RESULTS
Table 1 shows the socio-demographics characteristics of the women who participated in these study participants. About $44 \%$ of our participants were Muslims, followed closely by $27 \%$ of Christian Roman Catholic. Two thirds of participants were single at the time of the interview while less than a third were ever divorced or separated. About three quarter completed or never completed primary school while less than a quarter enrolled into a secondary school level. Very few were formally employed while the rest were either informally employed or unemployed.
\end{abstract}

Table 1. Socio-demographics characteristics of participants

\begin{tabular}{lllll}
\hline Age & $18-26$ & $27-36$ & $37-48$ & Total n (\%) \\
\hline $\begin{array}{l}\text { Number of participants in group } \\
\text { Place of Birth }\end{array}$ & $25(45 \%)$ & $20(36 \%)$ & $10(19 \%)$ & $55(100 \%)$ \\
Nairobi & & & & \\
Other urban areas & $8(60 \%)$ & $5(40 \%)$ & 0 & $13(23 \%)$ \\
$\begin{array}{l}\text { Rural area } \\
\text { Religion }\end{array}$ & $7(41 \%)$ & $9(52 \%)$ & $1(6 \%)$ & $17(31 \%)$ \\
Christian-Protestant & $6(24 \%)$ & $9(36 \%)$ & $10(40 \%)$ & $25(46 \%)$ \\
Christian-Roman Catholic & & & & \\
Muslim & $4(33 \%)$ & $5(42 \%)$ & $3(25 \%)$ & $12(22 \%)$ \\
Others & $6(40 \%)$ & $4(27 \%)$ & $5(33 \%)$ & $15(27 \%)$ \\
Marital status & $6(25 \%)$ & $8(33 \%)$ & $10(47 \%)$ & $24(44 \%)$ \\
Divorced/Separated & $3(75 \%)$ & 0 & $1(25 \%)$ & $4(7 \%)$ \\
Married & & & & \\
Single & 0 & $1(20 \%)$ & $4(80 \%)$ & $5(9 \%)$ \\
Education & $3(18 \%)$ & $6(35 \%)$ & $8(47 \%)$ & $17(31 \%)$ \\
Primary or less & $16(48 \%)$ & $13(39 \%)$ & $4(12 \%)$ & $33(60 \%)$ \\
Secondary & & & & \\
Tertiary & $18(45 \%)$ & $12(30 \%)$ & $10(25 \%)$ & $40(72 \%)$ \\
Employment & $2(18 \%)$ & $6(55 \%)$ & $3(27 \%)$ & $11(20 \%)$ \\
Formal & $2(50 \%)$ & $2(50 \%)$ & 0 & $4(7 \%)$ \\
Informal & & & & \\
Unemployment & $2(33 \%)$ & $4(67 \%)$ & 0 & $6(10 \%)$ \\
\hline
\end{tabular}

Most of our study participants revealed that they had had a sexual encounter in their lives. Some reported that they had unwanted pregnancies while others reported that they had procured unsafe abortions. Some of the key reasons why women procured unsafe abortions were due to stigma surrounding abortions, poverty and women's perceptions that abortion was still illegal and punishable in Kenya.

We present our findings in vignettes of two key participants that we name Caroline and Jane (pseudonyms). Caroline's experience demonstrates how unwanted pregnancies led to unsafe abortions and how these reflect the interrelationships 
between poverty, stigma, and religious beliefs. Jane's experience underscores how poverty and lack of knowledge on constitutional provisions of abortion in Kenya remains a major hindrance for women seeking safe abortion services.

Vignette 1: Caroline, 22years, lives in Kibera

Having been raised up in a rural set-up in western Kenya, Caroline was optimistic that one day, she would complete her secondary school education and move to Nairobi City to seek for a job. However, her dreams were never realized; at age 16 , she lost her parents. This forced her to move to Kibera informal settlements in Nairobi to live with her elder sister. Shenarrates her life in Kibera as follows:

"When I got to Kibera, life became more difficult because my sister who was the breadwinner in her family, stopped working immediately she delivered her second child. I was forced to start looking for casual jobs to be able to buy food; something that was too difficult for me."

She reported that the casual jobs were never promising because she could go for days without a job. As a result, she narrates;

"I started having sex with a man in my neighborhood for financial gains. This led to unwanted pregnancy. Unfortunately, when I told the man about it, he fled away".

Caroline narrated how she became more stressed, not knowing what to do with her unwanted pregnancy. She revealed how she was self-stigmatized for not being a good example in her church when she said;

"I was a very active member at our church. I felt like I had done something wrong."

Because Caroline was still young and financially unstable, she turned to her sister and a close friend foradvice, who in turn advised her to procure an abortion. She says;

“...they advised me to get some money and do abortion [..]. After a couple of weeks, I had accumulated about Ksh. 2000 (US\$2O. My friend willingly accompanied me to a private clinic in Kiber. The doctor was willing to help me. However, the clinic was so scary, it was dark with a small sized bed at the corner of the room, a black covered plastic container under the bed [...]. The doctor explained that if I made any noise, people will come in and both of us would be in trouble. He inserted a huge syringe with a sharp point through my vagina and I started feeling pain. The pain became unbearable, I started bleeding heavily. He gave me some heavy cotton to put in between my legs and some painkillers. I leftthe clinic and went back home; I barely slept that night. The following morning, I was too weak to even stand up. My sister called for help from a neighbor and I was rushed to Marie stopped clinic in Kibera. They were forced to pay a lot of money before I was attended to".

Caroline's case is not a unique one as more than three quarter of our study participants described howpoverty, stigma or lack of information on safe abortion services served as the major reasons why womensought abortion services from unsafe providers or self-induced abortions at home. It was reported that the cost of safe abortion services in Kibera ranged from Ksh. 6000 (US\$60) to Ksh.7000 (US\$70) in private hospitals or clinics such as Marie Stopes, something which in the context of financial insecurity is no small fee. Unsafe abortion services charged between Ksh. 300 (US\$3) -Ksh.1000 (US\$10) and was relatively affordable to most women. Additionally, participants indicated that they knew the dangers that 
were associated with unsafe abortions including method failure, over-bleeding and deaths; but they continued seeking abortion services from such providers:

"I know that I can die, but I just risk, I cannot afford safe abortion services." (A 35-year-old case study participant)

Accessibility and affordability factors were also said to override the risks and dangers associated with unqualified providers as reported by a 45-year-old community health worker:

"A good thing with the unsafe providers is that their prices are affordable, and they are easily available. They sell their concoctions in the open market".

Caroline's vignette further demonstrates that, both self and community stigma was linked to both unwanted pregnancies and abortion. First, women reported of self-stigma and shame when they realized that they had unwanted pregnancies. Second, those known to have aborted were also stigmatized at the community and this forced women to seek abortion services in a secretive manner, which risked their lives:

"There is a lot of stigma especially to a Muslim woman who is impregnated by a Christian; in Islam, this woman will be considered as an outcast." (35 years old woman).

"I will do it secretly and ensure that nobody knows about it otherwise, I will become a laughing stock and a topic for gossip". (20 years old woman).

Our study also revealed that most religions were against abortions. Interviews with our key informants from different religious backgrounds confirmed that abortion was condemned. One of our key informants, a 60-year-old protestant leader argued:

"Women are allowed to use contraceptives, but abortion is not considered as a contraceptive method but a sin".

Likewise, a 50-year-old Muslim head in one mosque in Kibera explained that:

"A woman has no right to kill a human being. That is Haram". (Haram is something forbidden or illegal).

Such religious stands against abortion drove women to procure abortions secretly from unsafe providers. However, even after procuring abortion, some women experienced mixed feelings of guilt, regret and shame as exemplified by an 18-year-old woman:

"I am a Christian-Roman Catholic and I believe that abortion is not good [...] I decided to procure abortion but when I think of it now, I feel that I committed a serious sin. It has been a bad experience for me and it has never escaped my mind that I killed an innocent soul"

\section{Knowledge of constitutional provi- sions on abortion in Kenya}

This study explored women's understanding of the provisions regarding abortion in the 2010 Kenyan constitution. The findings as indicated in table 2 revealed that about a third of the respondents were aware of what the constitution provided on abortion in Kenya. Most of them were educated up to secondary and tertiary levels. More than two thirds of women in this study lacked information about their rights to abortion. Majority of them were primary school drop outs. Such women were forced to seek abortion services from quacks since they were not able to afford abortion services from qualified providers. 
Journal of Maternal and Child Health (2019), 4(2): 97-109

https://doi.org/10.26911/thejmch.2019.04.02.05

Table 2. Predictors of knowledge of constitutional provisions on abortion in Kenya

\begin{tabular}{llll}
\hline \multicolumn{3}{l}{ Predictors of knowledge of constitutional provisions on abortion in Kenya (N=55) } \\
\cline { 2 - 3 } Predictors & \multicolumn{2}{c}{ Knowledge } & Total \\
\hline Age & $3(12 \%)$ & Poor & \\
$18-26$ & $5(25 \%)$ & $22(88 \%)$ & $25(54 \%)$ \\
$27-36$ & $2(20 \%)$ & $15(75 \%)$ & $10(18 \%)$ \\
$37-48$ & $10(18 \%)$ & $8(80 \%)$ & $55(100 \%)$ \\
& & $45(82 \%)$ & \\
Education & $4(10 \%)$ & $36(90 \%)$ & $40(73 \%)$ \\
Primary or less & $4(36 \%)$ & $7(64 \%)$ & $11(20 \%)$ \\
Secondary & $3(75 \%)$ & $1(25 \%)$ & $4(7 \%)$ \\
Tertiary & $11(20 \%)$ & $44(80 \%)$ & $55(100 \%)$ \\
& & & \\
Employment & $2(7 \%)$ & $28(93 \%)$ & $30(54 \%)$ \\
Unemployed & $3(16 \%)$ & $16(84 \%)$ & $19(35 \%)$ \\
Informal & $5(83 \%)$ & $1(7 \%)$ & $6(11 \%)$ \\
Formal & $10(18 \%)$ & $45(82 \%)$ & $55(100 \%)$ \\
& & &
\end{tabular}

This analysis leads to our second vignette which exemplifies how lack of knowledge on the legal provisions of abortion drove women to unqualified providers.

Vignette 2: Jane, 19 years, lives in Kibera

Jane, a first-born child in her family and primary school drop-out, narrates how poverty influenced her to sleep around with men for financial gains and when she became pregnant, she decided to procure unsafe abortion secretly to avoid being arrested by the police.

"I left school at age 14 to support my family. My father died when I was very young and my mother, who is HIV positive, has been providing for us until she became weak to continue working. I started working as a house maid in people's houses and was paid Ksh. 200 (US\$ 2) a day. This sustained my family for a while till the jobs started becoming scarce. I started going for days without getting any job and this compromised food provisions in my family. Some friends advised me to venture into prostitution which was said to be a lucrative business in Kibera. I did not hesitate, I started sleeping with men for money and I could earn about Ksh. 100-15o (US\$ 1-1.5) per client. Sometimes, men would have sex with me and refuse to pay but this was not so common. Unfortunately, I never thought of using contraceptives, and this led to unwanted pregnancy. Realizing this, I confided with oneof my friends, a middleaged woman who had introduced me into prostitution. She advised me to get an abortion immediately. Since abortion is illegal, my friend and I decided to look for backstreet providers who helped me induce the abortion. I went back home thinking everything was fine. After a week of abdominal pains and bleeding, I became weaker. My mother suspected that something was wrong; she confronted me and asked about it. I frankly opened up to her and said everything [...] she warned me from leaving the house lest I get arrested for having aborted. This kept me locked in the house for weeks, but the situation only worsened. At that point, my mother was 
forced to take me to Kenyatta hospital for treatment. This forced her to look for money for transportation and hospital procedures".

Jane's case represents many other women we interviewed who revealed that other than poverty and stigma, perceived illegality surrounding abortion in Kenya is also a major driving factor for women who seek abortion services secretly from unqualified providers. For example, during FGD discussions, most participants reported that they did not know the constitutional provisions on women wanting to terminate their pregnancies. Few women had a clue on what the constitutional provisions on abortion entailed but stated that abortion was only allowed "when the mother's health or that of the child is in danger", without mentioning other provisions such as'in the opinion of a trained health professional, in case of emergency or as provided by any other written law'. In one of the FGD session, one participant stated:

"I totally have no clue about constitutional provisions but what I know for sure is that abortion is illegal in Kenya”. (FGD 3, 18-24 years).

Another participant in a different FGD said:

"If the government could have legalized abortion, we could have heard it in the media [...] that means abortion is still illegal”. (FGD 1, 35-48 years)

Participants also reported that anyone who helped women procure abortion would be arrested and imprisoned. A 32-year-old case study participant said:

"The doctors are in danger too, if found helping a woman who wants to abort, he/shewill be arrested."

Yet, as revealed in Jane's case, some of the women who procured abortions from unsafe providers ended up in hospitals for post abortion care, which was said to be a costly procedure not only to the patient but also to the hospital. From the foregoing it appears that no sufficient awareness raising activities have been made to familiarize residents of Kibera on the new constitution and its provisions on abortion. As a result, most women, especially those of lower socio-economic status, still procure abortion from unqualified providers which expose them to post abortion complications.

\section{DISCUSSION}

This study explored on the factors influencing women's access to safe abortion services in Kibera informal settlements. Our findings showed that most women in our study have had unwanted pregnancies in their lifetime and some sought abortion services from unqualified providers. In addition, most participants seemed knowledgeable of the dangers of seeking abortion services from unqualified providers but due to the perceived illegality surrounding abortion, lack of financial support, and cultural and religious beliefs, they still sought their services. Our study concurs with earlier studies in Kenya which suggested that women and girls face numerous barriers to controlling their fertility, experience high rates of unintended pregnancy (Ikamari et al. 2013), commonly encounter stigma associated with unintended pregnancy and abortion (Centre for Reproductive Rights, 2010; Marlow et al., 2014; Izugbara et al., 2017; Mitchell et al., 2006), and resort to unsafe methods to end unwanted pregnancies (Izugbara et al., 2015; Izugbara et al., 2017; Mitchell et al., 2006).

While the Kenyan constitution of 2010 may have widened the legal provision under which abortion is permitted in Kenya (National Council for Law Reporting, 2010), it is evident from our study that a 
marked resistance to abortion still prevails at the community or societal level because; the government has not succeeded in removing the prevalence of anti-abortion sentiments among the public and also, it has not provided affordable and accessible safe abortion services especially amongst those living in lower socio economic settings.

This study revealed that most religious beliefs and doctrines were against abortion (safe or unsafe). They equated abortion to murder and such anti-abortion sentiments drove women to secretly procure abortions from unsafe providers. For the women who would defy such sentiments and go ahead to procure abortions, they were left with mixed feelings of guilt, shame and regret, a finding that has been reported in other settings (Shellenberg et al. 2011).

Furthermore, factors such as poverty, unemployment and lack of financial means limited women's autonomy to safe abortion services. For instance, most women in this study were unemployed, while others relied on unpredictable casual jobs within Kibera. This could be attributed to the fact that most were educated only up to primary levels, thus could not qualify for better jobs. This formed the basis why majority were not able to access or afford providers offering safe abortion services and this finding that has been reported elsewhere (Jayaweera et al. 2018; Ziraba et al. 2015; Sedgh et al. 2016).

The perceived illegality surrounding abortion in Kenya has the consequence of driving women to procuring abortions from unsafe providers. More than two thirds of our participants perceived abortion as illegal and this not only prevented women from seeking abortion care services from qualified providers but also contributed to abortion stigma and delayed care seeking after experiencing complications from induced abortions. Studies in other African countries have found that because of ignorance of the law, in the event of abortion complications, some women do not go to health facilities for treatment because of fear of prosecution by the law enforcement agencies (Muzeyen et al., 2017). Moreover, it has been argued that a woman's ability to obtain safe abortion services is affected not just by the law in a country but also by how these laws are interpreted and enforced and the attitude of the medical community toward abortion (Izugbara et al., 2015). The legalization of abortion is a necessary but insufficient step toward widespread access to safe abortion services and that for women to have access to safe abortion services, there is a need for them to know, interpret, and understand the laws pertaining to abortions in their countries (Sai, 2004; Hord and Wolf, 2004; Grimes et al., 2006).

The "three bodies" metaphor elucidates the problem of unsafe abortions in Kenya where widening of the legal framework pertaining to abortion in the 2010 Kenyan constitution may have minimized abortion restrictions at the macro-structural level; but cultural, economic and religious constraints at the individual and community level continue to exist and prove to be significant barriers to women's access to safe abortion. When making decisions regarding abortion, women in Kenya are left in a dilemma because of the varied meanings to abortion: the meaning surrounding abortion as morally wrong is in opposition to the meaning that the government derives from it, that is, to further its political ideology through the domain of reproduction and women's rights. Women's access to safe abortion services is also confined to meanings pertaining to affordability and accessibility 
of such services. These conflicting meanings clearly prove that comprehensive and appropriate sexual and reproductive choice and rights are yet to be achieved in Kenya.

Limitation to this study is that its results may not be generalizable to a larger Kenyan population, especially those in rural populations. However, our findings concur with other previous findings conducted in different contexts in Kenya and in other African countries and thus, we believe that the findings are transferable beyond the bounds of the immediate study population.

It is evident that poverty, religion, culture and stigma plays a significant role in women's access to safe abortion services in Kenya.Expansion of legal provisions of abortion in Kenya is good but not enough. We recommend that it is imperative for the Kenyan government to work towards not only creating awareness about what the constitution provides for safe abortion in Kenya but also by changing socio-economic and religious forces that still make access to safe abortions difficult or impossible for many women. In addition, abortion policy regulations should intentionally facilitate access to safe abortion services for all women, inform healthcare providers of their obligations in this regard, and inform women about the services to which they have a right.

\section{ACKNOWLEDGEMENTS}

We wish to thank all of those who participated in this study; their sincerity in sharing their life stories and personal experiences were fundamental to the success of this study. This research was funded through a Masters' degree scholarship awarded to the first author by the University of Nairobi through the Institute of Anthropology, Gender and African Studies.

\section{COMPETING INTERESTS}

None.

\section{REFERENCES}

Cameron C (2005). In the world of Dolly, when does a human embryo acquire respect?. Journal of Medical Ethics, 31(4): 215-220. doi: 10.1136/jme.2003.006395 .

Centre for Reproductive Rights (2010) In Harm's Way: The Impact of Kenya's Restrictive Abortion Law. New York.

Daily Nation (2018). Unsafe abortions kill seven Kenyans daily. https://www.nation.co.ke/news/Unsafe-abortionskill-seven-Kenyans-daily/1056-4769952-i6kd8pz/index.html.

Gleeson R, Forde E, Bates E, Powell S, Eadon-Jones E, Draper H (2008). Medical students' attitudes towards abortion: a UK study. Journal of Medical Ethics, 34(11): 783-787. doi: 10.1136/jme.2007.023416.

Grimes DA, Benson J, Sigh H, Romero M, Ganatra B, Okonofua FE, Shah IH (2006). Unsafe abortion: The preventable pandemic. The Lancet Sexual and Reproductive Health Series, 368(9550): 1908-1919.

Hord C, Wolf M (2004). Breaking the cycle of unsafe abortions in Africa. African Journal of Reproductive Health, 8(1): 29-34.

Hussain R (2012). Abortion and unintended pregnancy in Kenya. Issues Brief (Alan Guttmacher Inst).

Ikamari L, Izugbara C, Ochako R (2013). Prevalence and determinants of unintended pregnancy among women in Nairobi, Kenya. BMC Pregnancy and Childbirth. 13(1). https://doi.org/10.1186/1471-2393-13-69

Izugbara C, Ochako R (2011). Unwanted pregnancy and pregnancy termination among women in Nairobi, Kenya. 
Policy Brief, Nairobi, Kenya: African Population and Health Research Centre.

Izugbara $\mathrm{CO}$, Egesa $\mathrm{CP}$, Kabiru CW, Sidze EM (2017). Providers, Unmarried Young Women, and Post-Abortion Care in Kenya. Studies in Family Planning, 48(4): 343-358. doi: 10.1111/sifp.12035.

Izugbara CO, Egesa COR (2015). High profile health facilities can add to your trouble: Women, stigma and un/safe abortion in Kenya. Soc Sci Med. 141: 9-18.

Jackson M (1996). Things As They Are:

New directions in Phenomenological Anthropology. Indiana University Press: Bloomington.

Jayaweera RT, Ngui FM, Hall KS, Gerdts C (2018). Women's experiences with unplanned pregnancy and abortion in Kenya: A qualitative study. PLoS One. 13(1): e01. https://doi.org/\%oA10.1371/\%oAjournal.pone.\%oA0191412.

Kenya National Bureau of Statistics (KNBS), ICF M (2010). Kenya Demographic and Health Survey 2008-09. Calverton, Maryland: KNBS and ICF Macro.

Kumar A, Hessini L, Mitchell EMH (2009). Conceptualising Abortion Stigma. Culture, Health and Sexuality, 11:(6). https://doi.org/10.1080/1369105090 2842741.

Lee P (2004). The Pro-Life Argument from Substantial Identity: A Defence. Bioethics 18:3: 249-26.

Lopez R (2012). Perspectives on Abortion: Pro-Choice, Pro-Life, and What Lies in between. European Journal of Social Sciences, 27(4): 511-517. http://www.europeanjournalofsocialscienc es.com.

Marlow HM, Wamugi S, Yegon E, Fetters T, Wanaswa L, Msipa-Ndebele S (2014).
Women's perceptions about abortion in their communities: perspectives from western Kenya. Reprod Health Matters. 22(43): 149-58.

Mayi-Tsonga S, Assoumou P, Olé BS, Ntamack JB, Meyé JF, Souza MH, Faúndes A (2012). The contribution of research results to dramatic improvements in post-abortion care: Centre Hospitalier de Libreville, Gabon. Reproductive Health Matters, 20(40): 16-21.

Mitchell EM, Halpern CT, Kamathi EM, Owino S (2006). Social scripts and stark realities: Kenyan adolescents' abortion discourse. Cult Health Sex. 8(6): 515-28. Doi: 10.1080/13691050600888400

Muzeyen R, Ayichiluhm M, Manyazewal T (2017). Legal rights to safe abortion: knowledge and attitude of women in North-West Ethiopia toward the current Ethiopian abortion law, Public Health. Elsevier Ltd. 129-136. doi: 10.1016/j.puhe.2017.03.020.

National Coordinating Agency for Population and Development (2010). Maternal deaths on the rise in Kenya: A call to save women's lives. National Coordinating Agency for Population and Development ( NCAPD): Nairobi.

National Council for Law Reporting (2010). Constitution of Kenya. Nairobi, Kenya: NCLR with Authority of the Attorney General.

Ndunyu L (2013). Women's Experiences of Induced Abortion in Mombasa and Kilifi Districts, Kenya. PhD,University of KwaZulu Natal.

Sai F (2004). International commitments and guidance on unsafe abortion. African Journal of Reproductive Health, 8(1): 16-18.

Sawicki N (2011). Abortion Informed Consent Debate: More Light, Less Heat, 
The Cornell JL \& Pub. Pol'y. Available at: http://heinonlinebackup.com/holcgi-bin/get_pdf.cgi?handle $=$ hein.journals $/$ cjlpp21\&section $=4$. Scheper-Hughes N, Lock MM (1987). The Mindful Body: A Prolegomenon to Future Work in Medical Anthropology. Medical Anthropology Quarterly, 1(1): 6-41.

Sedgh G, Bearak J, Singh S, Bankole A, Popinchalk A, Ganatra B (2016). Abortion incidence between 1990 and 2014: global, regional, and subregional levels and trends. The Lancet. 388(10041): 258-267.

Shellenberg KM, Moore AM, Bankole A, Juarez F, Omideyi AK, Palomino N, et al. (2011). Social stigma and disclosure about induced abortion: results from an exploratory study.
Glob Public Health. 6(): S111-S25. Siegel RB (2014). Abortion and the "Woman Question": Forty Years of Debate. Indiana Law Journal, 89(4): 1365-1380. Available at: http://search.ebscohost.com/login.aspx?dire $c t=$ true \&db=a9h\&AN=96565112\&site $=$ ehost-live\&scope $=$ site.

Vélez ACG (2012). The health exception": a means of expanding access to legal abortion. Reprod Health Matters., 220(40): 22-9.

Ziraba AK, Izugbara C, Levandowski B, Gebreselassie H, Mutua M, Mohamed SF, Egesa C, Kimani-Murage EW (2015). Unsafe abortion in Kenya: Across-sectional study of abortion complication severity and associated factors. BMC Pregnancy and Childbirth, 15(1):34. 